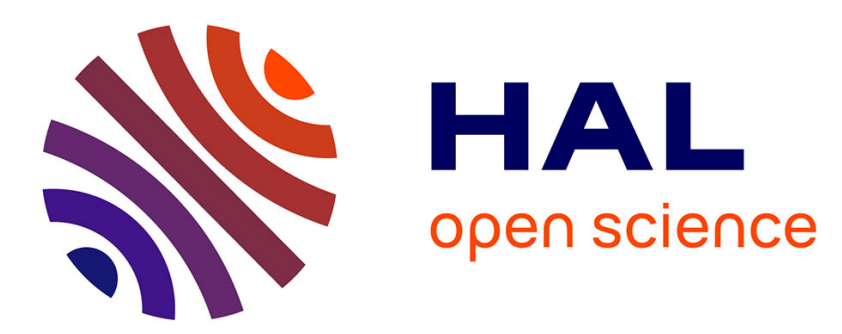

\title{
Femmes, droits et participation citoyenne au Machrek, au Maghreb et en Turquie
}

Nora Semmoud, Gülçin Erdi, Safaa Monqid

\section{To cite this version:}

Nora Semmoud, Gülçin Erdi, Safaa Monqid. Femmes, droits et participation citoyenne au Machrek, au Maghreb et en Turquie. Genre, féminismes et développement. Une trilogie en construction, 2019. halshs-02166441

\section{HAL Id: halshs-02166441 https://shs.hal.science/halshs-02166441}

Submitted on 26 Jun 2019

HAL is a multi-disciplinary open access archive for the deposit and dissemination of scientific research documents, whether they are published or not. The documents may come from teaching and research institutions in France or abroad, or from public or private research centers.
L'archive ouverte pluridisciplinaire HAL, est destinée au dépôt et à la diffusion de documents scientifiques de niveau recherche, publiés ou non, émanant des établissements d'enseignement et de recherche français ou étrangers, des laboratoires publics ou privés. 
Nora Semmoud, Gulçin Erdi et Safaa Monqid, 2019, Chapitre 15 « Femmes, droits et participation citoyenne au Machrek, au Maghreb et en Turquie ", in Chairman Levy, Andrea Martinez (dir.), Genre, féminisme et développement. Une trilogie en construction, Les presses de l'Université d'Ottawa, pp. 295-312, 501 p.

\section{Résumé}

Dans ce chapitre, il est question d'analyser les droits des femmes et les formes spécifiques de leurs engagements, avec l'ambition de contribuer à une meilleure connaissance de la condition féminine des musulmanes en Turquie et dans plusieurs pays du Machrek et du Maghreb ; une réalité souvent tronquée par un certain regard colonial qui occulte les transformations que connaissent ces sociétés. Tout en soulignant les particularités des différents pays, la réflexion met en évidence les conditions historiques d'émergence et d'évolution de la question de l'émancipation des femmes et de leurs droits. Un processus qui s'avère long et difficile, depuis les affres du colonialisme et de la guerre jusqu'à la montée récente du conservatisme et de l'islamisme, en passant par les effets paradoxaux du développement. Il s'agit plus particulièrement de rendre compte, un tant soit peu, des voies de l'émancipation des femmes et de leur mouvement, en contextes difficiles, mais aussi des hybridations qu'elles opèrent en s'appropriant notamment les modèles internationaux et en les réinterprétant au sein de leurs sociétés. Le propos est structuré en trois sections. Dans la première, les auteures retracent l'histoire de la question féminine, en analysant les raisons des avancées et des reculs. Dans la deuxième section, elles focalisent sur les droits des femmes et sur leurs luttes pour les maintenir et les faire progresser face à une pensée patriarcale et conservatrice fondée sur une interprétation rigoriste de l'islam. Enfin dans la troisième section, les auteures centrent leur réflexion sur les facteurs qui influent actuellement dans l'évolution/régression du mouvement des femmes.

\section{Concepts-clés}

Droits des femmes, Féminismes, Islamisme, Laïcité, Luttes anticoloniales

\section{Introduction}

La vague du mouvement protestataire dans les pays du monde arabe et en Turquie a surpris le reste du monde par son ampleur et ses résultats, mais aussi par la présence importante des femmes. Amel Mahfoudh et Christine Delphy (2014 : 4) attribuent cet effet de surprise à la persistance du regard néocolonial dans de nombreux pays pour lesquels les transformations sociétales de ces contrées sont un champ aveugle (Lefebvre, 1970 : 44). S'écartant des poncifs, l'objectif du présent chapitre est de permettre une meilleure connaissance de la condition des femmes et de leur mouvement au Machrek, au Maghreb et en Turquie et, plus largement, une compréhension de ces sociétés. Il s'agit également de montrer les voies particulières de l'émancipation des femmes et les hybridations qu'elles opèrent en s'appropriant les modèles internationaux, en lien avec la réalité de leurs sociétés. Les questionnements suivants structureront la réflexion : quel est l'ancrage historique des dynamiques d'émancipation des femmes au Machrek, au 
Maghreb et en Turquie, et quels sont les éléments déclencheurs? Quelles sont les conditions d'émergence de leurs droits? Comment, face aux facteurs de régression (tensions politiques et guerres; montée du conservatisme islamiste), les femmes poursuivent-elles leur processus d'émancipation? Les concepts utilisés dans ce travail ont autant trait à la condition des femmes, comme leur mouvement, leurs féminismes ou leurs droits, qu'aux contextes dans lesquels elle s'inscrit, comme la laïcité, la montée de l'islamisme conservateur et/ou radical, les luttes anticoloniales, le nationalisme, l'empowerment ou l'espace public.

Soulignons que cette réflexion, loin d'être exhaustive, constitue une synthèse qui a pour seule prétention de susciter la réflexion et d'encourager à poursuivre les lectures sur les questions soulevées. En outre, les pays étudiés (voir la figure $1^{1}$ ) - l'Égypte, la Syrie, la Jordanie et la Palestine pour le Machrek; le Maroc, l'Algérie et la Tunisie pour le Maghreb - ne représentent pas toute la diversité de la réalité des femmes de ces contrées, a fortiori pour un grand territoire comme la Turquie.

Figure 1. Aire étudiée.

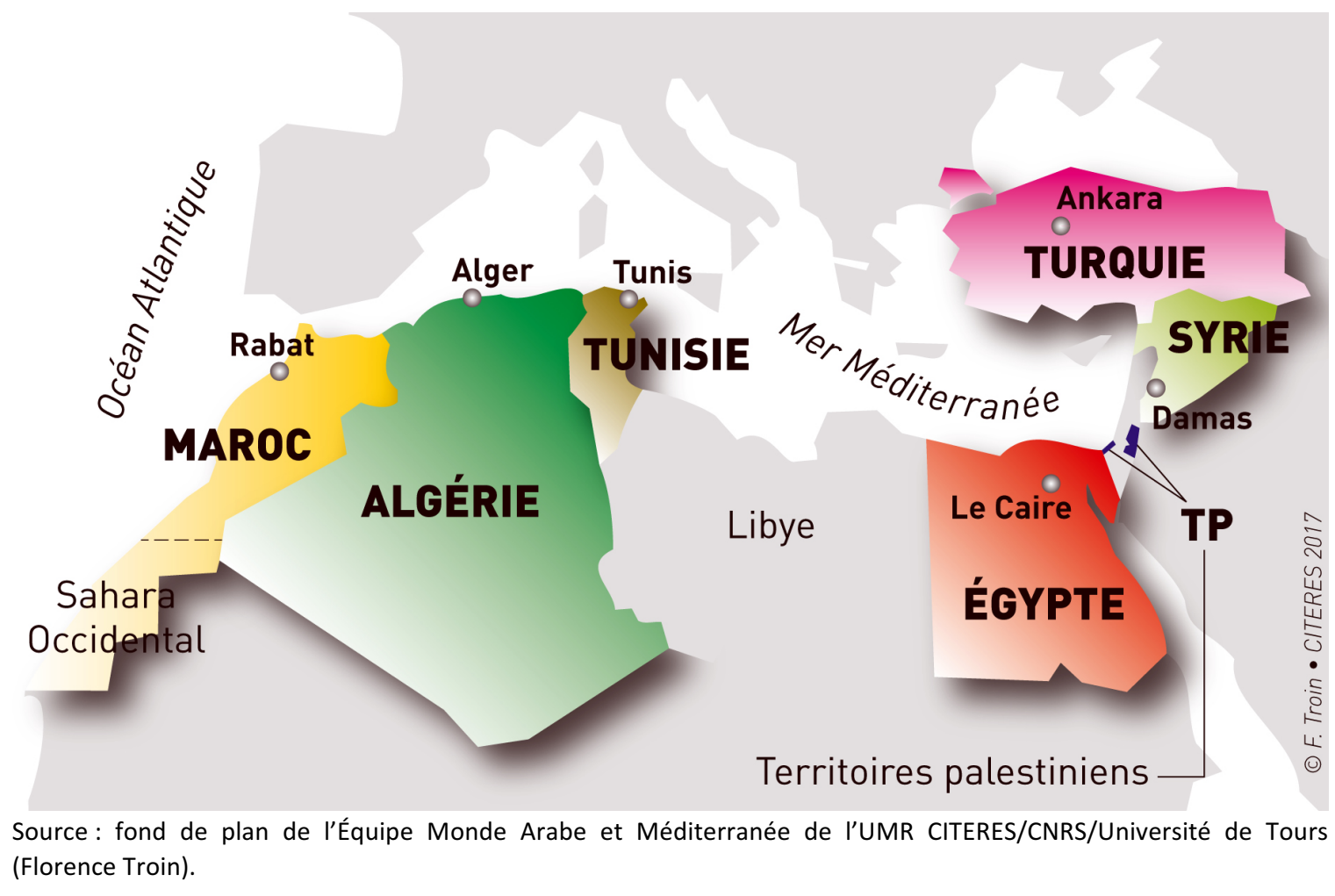

\section{Histoire de la question féminine au Machrek, au Maghreb et en Turquie}

Jusqu'à la fin du XIX ${ }^{\mathrm{e}}$ siècle, les espaces publics dans les pays du Machrek et du Maghreb de même qu'en Turquie étaient généralement réservés aux hommes, et de fortes dichotomies spatiales recoupaient la ségrégation sexiste. Les femmes étaient assignées à l'espace domestique pour des raisons religieuses, culturelles et sociales. Seules la 
nécessité et la pauvreté pouvaient justifier qu'une femme sorte de son foyer. Ces sociétés, dont les rapports étaient structurés par le patriarcat et ses valeurs conservatrices, ont ensuite subi des bouleversements liés aux luttes nationalistes et anticoloniales, provoquant des évolutions notables dans la condition des femmes.

De la fin du XIX ${ }^{\mathfrak{e}}$ siècle jusqu'au début du $\mathrm{XX}^{\mathrm{e}}$ siècle, on assiste, au Proche-Orient, au déclin de l'Empire ottoman et à l'établissement des mandats français sur la Syrie et le Liban, et des mandats anglais sur la Palestine, l'Iran, l'Irak, la Transjordanie et l'Égypte. La création de nouveaux États a permis la mise en place de réformes économiques, sociales et politiques. Elle a en outre favorisé l'apparition de nouveaux acteurs : une jeunesse nationaliste avide de changement et des femmes en quête d'émancipation. Cette période a été marquée par la fin des harems et l'émergence de la question féminine, grâce au mouvement de la Renaissance féminine de l'entre-deux-guerres. Soulignons que l'écrivain Jurgi Zaydan (1861-1914) fut le premier à utiliser le mot nahda, qui fait référence à la Renaissance européenne, en rupture avec l'obscurantisme du Moyen Âge (asr al-inhitât). Dans ce contexte, le nationalisme considérait les femmes comme un maillon central de la construction de la nation et encourageait leur apparition dans la vie publique. Grâce au travail et au salariat, elles ont entamé une conquête de la ville favorisée par de nouveaux espaces publics (promenades de bord de mer, salles de spectacles, jardins publics, etc.) et par la levée, en grande partie, des interdits. Elles se dévoilèrent peu à peu, en ôtant d'abord le voile de leur visage (fin des années 1920 pour les Égyptiennes et les Turques). En Syrie et au Liban, le costume européen se généralisa chez les femmes dès la fin du XIX ${ }^{\mathrm{e}}$ siècle, bien qu'elles gardèrent le voile jusqu'en 1900. Leur adhésion à cette dynamique n'avait cependant pas été univoque en raison de la diversité de leurs profils (citadines, rurales) et de leurs conditions sociales (les riches citadines étaient recluses, tandis que les femmes des couches défavorisées travaillaient), mais aussi à cause de la pluralité des confessions. En Syrie et au Liban, par exemple, il y avait, au XIX ${ }^{\mathrm{e}}$ siècle, une grande différence de scolarisation entre les chrétiennes et les musulmanes. Les premières ont pu intégrer très tôt les écoles mises en place par les missionnaires, tandis que les secondes ont franchi le pas bien plus tardivement.

Si l'amélioration de la condition féminine était un enjeu important pour les réformistes de l'époque, qui dénonçaient les effets néfastes de la claustration des femmes et considéraient qu'elles devaient s'instruire afin d'éduquer les générations futures, les autres discours variaient selon les mouvances. En Égypte, on distinguait les idées des progressistes, comme celles de Qásim Amín (1863-1908) connu pour ses positions courageuses dans les ouvrages La libération de la femme, paru en 1899, et La femme nouvelle, paru en 1900; les optiques conservatrices, représentées par Malak Hifni Nasef (1886-1918), qui prônaient l'amélioration de la condition féminine dans le cadre strict de la religion; et, enfin, les postures rigoristes portées par Zaynab al-Ghâzâli (1917-2005), dirigeante du mouvement islamique des femmes, à l'origine de l'association des Sœurs musulmanes en 1935. Ces clivages ont sans doute été suscités par l'accès des femmes à la vie publique encouragé par la mobilisation des nationalistes. Ainsi, de nombreuses femmes ont participé aux luttes pour la décolonisation, marquant la scène publique de 
leur présence. En Égypte, plusieurs d'entre elles ont manifesté lors de la révolution de 1919, menée par Sa‘d Zaghlûl, contre la colonisation britannique; parmi elles, Hudá Sharáwí (voir la figure 2), personnalité marquante du féminisme égyptien, présidente du comité des femmes du Wafd (le plus ancien parti nationaliste), qui a dirigé le mouvement féminin nationaliste entre 1919 et 1923.

Figure 2. Hudá Sharáwí, dirigeante du mouvement féminin égyptien entre 1919 et 1923

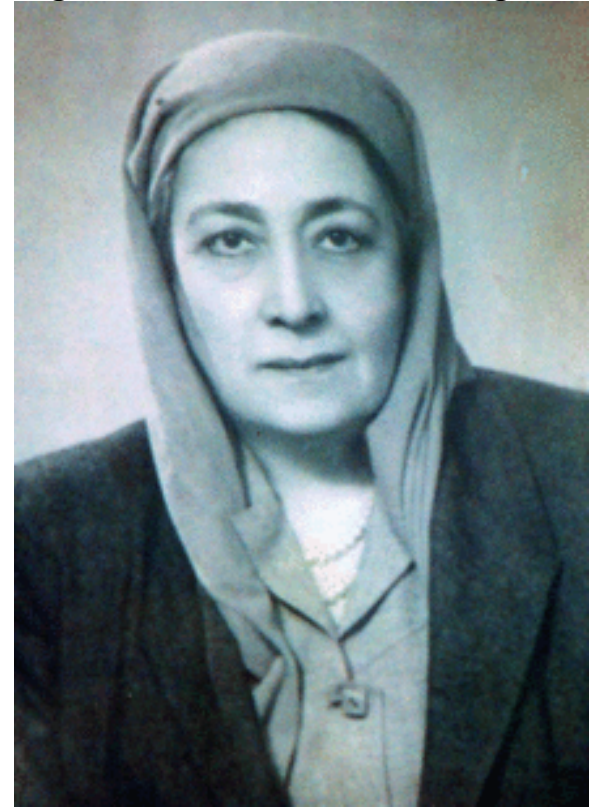

Source : Wikipedia.org (photographe inconnu ; domaine public)

Grâce à son histoire et à son poids démographique, culturel et religieux, l'Égypte occupa une place importante dans le mouvement d'émancipation des femmes du monde arabe et constitua un modèle de la Renaissance féminine. Plus grande ville du monde arabe, moderne, cosmopolite et pionnière dans bien des domaines, le Caire avait accueilli une importante communauté syrienne, à l'origine d'un mouvement de presse arabe qui a joué un grand rôle dans la diffusion des idées de la $n a h d a^{2}$ (mouvement de renaissance arabe). En effet, les premiers journaux, tels qu'Al-fatât (1892) et Fatât ash-sharq (1906), ont été fondés par des SyrienNEs chrétienNEs installéEs en Égypte pour fuir la répression ottomane. En Égypte et dans l'ensemble des pays du Levant, la presse arabe a été un moyen d'expression des femmes dans les débats sur leur accès à l'éducation et au travail ainsi que les conditions du mariage.

En Syrie et au Liban, le mouvement de la Renaissance féminine (An-nahda an-nisâ' 'iyya) a été amorcé dès le XIX ${ }^{\mathrm{e}}$ siècle par les missionnaires protestants, catholiques et d'autres communautés (grecques, arméniennes, juives, etc.), qui ont fondé les premiers établissements scolaires et universitaires ouverts aux femmes d'autres confessions, et par l'action des élites locales, en majorité chrétiennes, comme Ahmad Fâres as-Shidyâq (1809-1887) et Butros al-Bustânî (1819-1883). L'Irak indépendant de Fayçal n'était en reste ni en matière d'instruction des femmes ni du point de vue de leur mobilisation dans les luttes pour l'indépendance. Les Irakiennes ont participé au mouvement nationaliste durant les années 1920 et 1930. Elles ont créé leur première association en 1923, 
l'Association de la renaissance féminine, qui a œuvré pour l'accès à l'éducation et au travail et pour le droit de vote, mais qui s'est aussi engagée dans le travail social (Arenfeldt et al., 2012 : 13). À l'instar d'autres contextes sociétaux, la Première Guerre mondiale a accéléré les transformations des conditions des femmes levantines, qui se sont imposées comme chefs de famille du fait de l'absence des hommes, et qui ont été actives dans la prise en charge des orphelinEs. Des femmes de tout bord, chrétiennes et musulmanes, ont pris part ensuite au mouvement de libération; elles ont adhéré aux idées et aux actions indépendantistes et nationalistes de Fayçal.

En Jordanie, le mouvement des femmes s'est limité au travail social jusqu'en 1945, alors que fut créée l'Union des femmes jordaniennes, dont les revendications portaient sur leurs droits politiques, économiques et sociaux. Mais la proximité de ses dirigeantes avec la famille royale a nui à son élargissement. Étroitement lié aux luttes politiques, le combat des Palestiniennes a fait naître un réseau de solidarité des femmes arabes, à l'origine de diverses rencontres comme le Congrès féministe arabe du Caire, en décembre 1944 (Jasser et al., 2016). Dans les années 1970, les Palestiniennes ont réorienté leurs actions vers les organisations estudiantines ou syndicales, en établissant progressivement leurs propres structures pour défendre leurs droits.

L'incursion historique sera plus brève pour le Maghreb, car l'émergence du mouvement des femmes est devenue tangible pendant les luttes anticoloniales. De la même façon que les femmes du Machrek, les Maghrébines ont été particulièrement présentes dans le combat contre le colonialisme, dans les mouvements d'indépendance et dans la reconstruction nationale postindépendance (pour le Maroc, Benadada, 1999; pour la Tunisie, Mahfoudh et Mahfoudh, 2014; pour l'Algérie, El Korso, 1998).

En tant qu'État laïc, la Turquie a fait figure d'exception parmi les pays islamiques. L'histoire turque montre que la "question de la femme » était un élément majeur des réformes nationales dès la fondation de la République et que les Turques ont été les premières musulmanes à obtenir des droits juridiques et sociaux, notamment avec leur participation directe aux élections en 1934. Au Maghreb, le droit de vote des femmes a été soit consécutif, soit parallèle aux indépendances (Tunisie en 1959, Algérie en 1962 et Maroc en 1963). Au Machrek, la Syrie et l'Égypte l'ont mis en place respectivement en 1953 et en 1956, tandis que dans les autres pays, cet acquis a été plus tardif (Jordanie en 1974, Irak en 1980). De plus, la Turquie moderne a hérité d'une histoire riche de traditions multinationales et multiculturelles. Aujourd'hui, le statut des Turques n'en est pas moins complexe et il varie très largement, allant de cadres hautement qualifiées à une majorité de femmes peu diplômées. Il existe des différences importantes dans le mode de vie, non seulement entre les femmes urbaines et rurales, mais aussi entre celles d'une même classe sociale, selon leur niveau d'éducation et leur statut en matière d'emploi.

Les luttes des femmes pour revendiquer et renforcer leur émancipation en Turquie ont été forgées dans le cadre de l'émergence et de la consolidation de la République laïque au début du $\mathrm{XX}^{\mathrm{e}}$ siècle, après l'effondrement de l'Empire ottoman. Rappelons qu'après avoir perdu la Première Guerre mondiale, menée aux côtés des Allemands, une partie des 
insurgés de l'armée ottomane, avec à leur tête Mustafa Kemal Atatürk, ont organisé la résistance en Anatolie face à l'invasion des pays européens vainqueurs. Cette guerre de libération a débouché sur la fondation de la République de Turquie, en 1923. En raison de sa longue durée (1914-1922) et d'une diminution sensible de la population masculine, la jeune République a accordé une importance particulière à la formation des femmes et leur a attribué un rôle d'éducatrices. De nombreuses écoles pour filles ont été ouvertes ainsi que des « écoles normales » pour former des professeures.

Dans l'élan de la fondation de la jeune République, les Turques ont même fondé le Parti populaire des femmes afin de revendiquer des droits politiques et sociaux. Mais ce parti politique a été très vite interdit pour laisser place à l'Union turque des femmes qui organisera, en 1934, la première manifestation féminine à Ankara, capitale de la nouvelle République. Cette manifestation a débouché sur la promulgation, par le Parlement, des droits de vote et d'exercice de fonctions électorales pour les Turques. Fort de cette victoire, le mouvement des femmes s'est entièrement consacré à surveiller le traitement de la question féminine dans les réformes républicaines et laïques. Cette adhésion quasi idéaliste à l'optique républicaine et laïque s'est poursuivie jusque dans les années 1970. Ensuite, la montée en puissance des partis politiques proches des idées socialistes, avec leurs leaders féminines, a donné une nouvelle direction au mouvement des femmes. Parmi les leaders féminines, citons Behice Boran qui, à la tête du Parti des ouvriers dans les années 1970, a contribué à la diffusion des idées féministes dans le cadre de l'idéologie socialiste. Cependant, après le coup d'État militaire de 1980, le mouvement des femmes s'est progressivement éloigné des courants politiques socialistes en s'autonomisant davantage et en formant une dynamique féministe proche de ses homologues européennes.

Tant dans les régions du Machrek et du Maghreb qu'en Turquie, les femmes mobilisées pour l'indépendance de leur pays - et apprenant de cette mobilisation - défendaient en parallèle leurs droits, notamment le droit à l'éducation, au vote, et à des lois justes sur le mariage et le divorce. Les luttes des femmes pour leurs droits se sont alors naturellement poursuivies au lendemain des indépendances. Ainsi, les villes du Machrek ont été le théâtre de grandes mobilisations pour l'émancipation des femmes. Or, la plupart de ces mouvements ont été récupérés par les États arabes, et les actions des femmes ont été cantonnées aux œuvres caritatives. En Égypte, Suzanne Mubarak fonda le Conseil national des femmes (NCW), qui monopolisa toutes les actions de défense de leurs droits. En Syrie, dès 1963, le parti Ba'th institutionnalisa les mobilisations sociales par la mise en place d'organisations populaires et par la création, en 1967, de 1'Union générale des femmes de Syrie. Celle-ci avait pour mission de promouvoir «officiellement» leur statut, tandis que la mise en place d'autres associations de défense des droits des femmes était interdite (Lavoix, 2010). De même, en Irak, à l'exception de l'Union des femmes irakiennes, créée en 1968 par le régime, toutes les autres organisations féminines ont été interdites. On a assisté ainsi à plusieurs formes d'instrumentalisation de la question des femmes par les États à des fins politiques, pour renforcer la légitimité de leurs régimes et pour redorer leur image sur la scène internationale. 
De la même façon, dès que le Maghreb a obtenu son indépendance, les dynamiques qui affirmaient une volonté de promouvoir des revendications spécifiques aux femmes et une autonomie d'organisation ont rencontré de fortes oppositions. Jusqu'à la fin des années 1970, les organisations officielles ont monopolisé et dénaturé les questions féminines, tandis que les initiatives autonomes des femmes étaient généralement contrariées, contrôlées et réprimées. Selon les contextes, les gouvernements successifs ont oscillé entre des politiques d'émancipation visant les femmes et une tendance au conservatisme, notamment concernant leurs droits juridiques. En Algérie, par exemple, l'État a mis en place des politiques importantes pour la scolarisation des filles et pour l'égalité salariale entre les femmes et les hommes, tandis qu'en matière de droits juridiques, la vision demeurait rétrograde.

Malgré ces contradictions au sein des États, la logique volontariste du développement dans ces différents pays a permis d'améliorer de façon tangible la condition des femmes. Leur accès à la scolarisation et à l'emploi, conjugué à l'urbanisation et à l'amélioration des niveaux de vie, plus particulièrement dans les milieux citadins, a contribué à leur promotion, mais aussi, plus généralement, aux transformations sociétales comme le recul de l'âge du mariage, la limitation des naissances, la nucléarisation des familles, etc. Les femmes sont apparues comme des actrices incontournables du développement et comme un élément important de la modernisation des sociétés, même si ces dynamiques restaient contrariées par des règles et des normes sociales conservatrices.

Dans les années 1980, des facteurs nationaux comme l'accès des femmes à l'éducation ${ }^{3}$, à la formation, au travail et au planning familial ${ }^{4}$, mais aussi une relative liberté d'expression se conjuguent à des facteurs internationaux comme les programmes issus des conférences mondiales sur les femmes. Le tout se traduit par l'éclosion, dans les pays arabes, d'un important tissu associatif féminin en rupture avec les organisations officielles, qui relance les revendications pour l'émancipation et l'égalité des droits. Ces associations émanent d'élites féminines, généralement de gauche, parfois déjà impliquées dans des partis ou des syndicats.

\section{Des droits obtenus de haute lutte}

Depuis les années 1980, on assiste, dans les pays étudiés, à la résurgence d'une pensée patriarcale et conservatrice qui s'appuie sur une interprétation rigoriste de l'islam. Présente dans toutes les sphères de la société, cette pensée fait pression sur les pouvoirs en place pour remettre en cause les droits des femmes et pour instituer un modèle inspiré par la charia. Dans ce contexte difficile, les progrès en matière d'émancipation des femmes sont particulièrement laborieux et chaque acquis est obtenu de haute lutte.

\section{Les droits juridiques : un nœud gordien}

Les droits juridiques des femmes dans le monde arabe et musulman constituent par excellence le champ de cristallisation des luttes contre le conservatisme. Au Machrek, les 
codes du statut personnel qui ont vu le jour dans le contexte d'accaparement de la question féminine par les États ont déçu les espoirs des femmes. Les lois relevant de la logique patriarcale ont ainsi été reconduites, institutionnalisant l'inégalité entre les sexes et la soumission des femmes à l'autorité du père et de l'époux. Conscientes de ce mécanisme institutionnel d'exclusion, les femmes se sont mobilisées pour changer ces codes et ont créé, dans ce but, plusieurs associations comme l'Alliance of Arab Women (1987) ou New Woman, (1991) en Égypte. Ces luttes ont progressivement fait évoluer l'arsenal juridique égyptien, comme la loi de 1979 qui garantissait aux femmes le droit au divorce et celui de continuer à vivre dans la maison familiale tant qu'elles avaient la garde des enfants. En 2004 et 2005, de nouvelles lois ont permis de prolonger la garde des enfants jusqu'à 15 ans et d'établir un accès équitable et rapide à la pension alimentaire. En 2008, un nouveau texte sur les mineurEs relève l'âge du mariage de 16 à 18 ans et criminalise les mutilations génitales féminines. La Constitution égyptienne de 2014 a été présentée comme une avancée exceptionnelle pour les droits des femmes puisqu'elle institue l'égalité avec les hommes dans les fonctions électives et les postes clés de la fonction publique. Dans la même optique, une loi promulguée criminalise le harcèlement sexuel et donne, par ailleurs, le droit à une mère égyptienne de transmettre sa nationalité à ses enfants.

$\mathrm{Au}$ Maghreb, le processus pour faire évoluer les droits des femmes dans la famille a été plus ou moins laborieux, selon l'histoire et le système politique de chaque pays. Au Maroc, après l'indépendance, le Code de la famille était très conservateur, car la monarchie s'appuyait sur l'ordre tribal, hostile à la modernisation des lois. Les luttes des Marocaines pendant plusieurs décennies aboutiront finalement, en 2004, à la modification du Code de la famille (la Moudawana) afin de supprimer le devoir d'obéissance des épouses et d'introduire la coresponsabilité familiale et l'égalité de l'âge au mariage. Le pouvoir tribal et conservateur en Tunisie étant moins influent qu'au Maroc, ce pays avait adopté, lors de son indépendance, un régime républicain moderne qui a produit le Code du statut personnel (1956) le plus avancé du monde arabe. Depuis les années 1920, le mouvement féministe tunisien est un acteur clé de cette victoire (Mahfoudh et Mahfoudh, 2014). Les élites politiques tunisiennes s'étaient appuyées sur le travail de théologienNEs réformateurs et réformatrices : leur interprétation ouverte du texte sacré les a conduitEs à interdire la polygamie et la répudiation, mais aussi à introduire le divorce judiciaire et à institutionnaliser le mariage comme un contrat civil avec possibilité de clauses particulières (communauté des biens, respect du droit au travail de l'épouse, suppression de la tutelle et de la contrainte matrimoniale pour choisir librement son conjoint). Les insuffisances du texte portent sur l'absence de l'égalité successorale et la nonreconnaissance des unions entre Tunisiennes (musulmanes) et non-musulmans.

En Algérie, le Code de la famille promulgué en 1984, considéré comme une infamie par le mouvement féministe, se révèle être une régression par rapport à la réalité des femmes au travail, en formation et dans la société (Saadi, 1991). Ce texte, et plus généralement la question des femmes, ont souvent été instrumentalisés dans les luttes opposant le pouvoir 
algérien aux islamistes radicaux (luttes qui se sont soldées par une guerre civile meurtrière entre 1991 et 2002). Le Code de la famille algérien a finalement été modifié en 2005, mais il considère encore les femmes comme des mineures. L'Algérie a récemment promulgué une loi (29 décembre 2016) qui criminalise les violences contre les femmes et le harcèlement sexuel. Cette loi punit toute forme d'agression, de violence verbale et psychologique ou de maltraitance. L'Algérie devient ainsi le deuxième pays du Maghreb, après la Tunisie, à criminaliser les violences envers les femmes. Au Maroc, un projet de loi similaire est à l'étude et fait l'objet de vifs débats avec les conservateurs.

En Turquie, la force du mouvement féministe a permis d'avoir un arsenal juridique plus dense et plus complet que dans le monde arabe. Selon les données de 2013 de la Banque mondiale, les lois sanctionnant la violence domestique, la violence physique et sexuelle et le harcèlement sexuel ont été mises en place par des réformes successives du Code pénal, la dernière ayant eu lieu en 2004. La Constitution renferme une clause de nondiscrimination sexiste et d'égalité devant la loi, notamment en ce qui a trait à l'héritage. Cependant, dans la vie quotidienne, la situation difficile de nombreuses Turques reflète les obstacles entre cet arsenal législatif et son application effective. Elles ont de plus en plus de difficulté à bénéficier du droit à l'avortement, notamment dans les hôpitaux publics. En 2015, elles consacraient quatre heures et dix-sept minutes par jour à l'entretien de leur maison, soit l'un des taux les plus élevés des pays de l'Organisation de Coopération et de Développement Économiques (OCDE). Enfin, 35,5\% des Turques déclarent avoir subi des violences physiques de la part de leur conjoint ou concubin.

\section{Accès aux fonctions électorales et politiques: entre volontarisme étatique et retard sociétal}

Alors que l'investissement du champ politique par les femmes turques est une tradition qui remonte aux années 1930, ce phénomène est relativement récent au Machrek et au Maghreb : selon les pays, il s'échelonne des années postindépendance aux années 2000. C'est en Syrie qu'ont été nommées les premières femmes ministres (Najat al Attar, ministre de la Culture en 1976) et procureures générales du monde arabe (1973-1974). L'Égypte avait été, en 1957, le premier pays de la région à élire des femmes au Parlement. En 1979, en Syrie, un décret présidentiel réserva 30 sièges parlementaires aux femmes; 35 seront élues, occupant ainsi $9 \%$ des 382 sièges. De même, la modification de la Constitution égyptienne (2007) visant à établir la politique des quotas de femmes au Parlement permettra, en 2009, de leur octroyer $12 \%$ des postes à pourvoir (BernardMaugiron, 2017). Il en est de même en Jordanie, où un système de quotas, adopté en 2003, a permis aux femmes d'obtenir 6 sièges sur 110, soit 5,4\%; ce quota a doublé en 2010 pour faire passer la représentation féminine à 10,9\%. En juillet 2011, une nouvelle loi sur les municipalités a fait passer le quota des femmes au sein des conseils municipaux de $20 \%$ à $25 \%$.

Les quotas ont été mis en place plus tardivement dans les pays du Maghreb, mais ce volontarisme des États ne suffit pas à combler les lacunes en matière de participation des 
femmes à la vie politique. Dans beaucoup de pays du Proche-Orient et du Maghreb, elles restent sous-représentées dans les processus de décision politique, en raison de barrières surtout sociales et culturelles. En Égypte, par exemple, malgré l'adoption de la Constitution de 2014 qui institue l'égalité entre les sexes, une seule femme a été nommée gouverneure d'une province ou membre du Conseil d'État, ce qui soulève des questions sur la portée effective des dispositions constitutionnelles.

\section{Le travail, un facteur d'émancipation contrarié?}

La présence de plus en plus grande des femmes dans le monde du travail est une réalité tangible dans les régions du Machrek et du Maghreb, et à fortiori en Turquie. Cependant, là encore, malgré des changements politiques et sociaux d'envergure, les inégalités de genre persistent et les discriminations au travail, en matière de statut, de salaire et d'accès à la formation et aux promotions restent importantes. Même lorsqu'elles sont cadres, les femmes ont des salaires inférieurs à ceux de leurs collègues masculins de même grade (selon la Banque mondiale, en 2004, les écarts de salaire étaient de l'ordre de $15 \%$ à $20 \%$ ) et elles n'accèdent que rarement aux postes de responsabilité. De plus, une majorité de femmes dans ces pays sont cantonnées au travail informel, où elles sont peu payées et non protégées. Ici, notre analyse concernera surtout le Maghreb, sachant que la situation des travailleuses au Machrek et en Turquie est sensiblement différente. Cependant, le contexte de mondialisation et de néolibéralisation des économies nous conduit à envisager des effets similaires sur le travail des femmes dans les différents pays étudiés, notamment l'augmentation de la précarité, du chômage et du travail informel.

Malgré les particularités de l'histoire politique, économique et sociale de chaque pays du Maghreb, les tendances en matière de travail féminin se recoupent. La transition démographique, l'accès à l'éducation et à la formation (la présence des filles aux niveaux secondaire et supérieur est aujourd'hui plus importante que celle des garçons), et l'évolution des représentations sociales à l'égard des travailleuses ont certes fait évoluer leur présence dans le monde du travail. Mais cette dynamique reste contrariée par les tendances à la régression du travail salarié et stable, à l'augmentation du travail précaire et informel, et à la montée du chômage et de la pauvreté. De plus, à partir des années 1980, les politiques économiques - privatisation, libéralisation des prix et ouverture aux marchés internationaux - mises en cuvre sous l'injonction des bailleurs de fonds internationaux dans les trois pays du Maghreb ont entraîné des tensions particulièrement vives pour les travailleuses.

Après avoir été parmi les plus bas au monde dans les années 1960, les taux d'emploi chez les femmes révèlent, depuis une trentaine d'années, dans les trois pays du Maghreb, une évolution de la participation des femmes au marché du travail, avec une croissance plus forte en milieu urbain. Au Maroc, la part des femmes dans la population active est passée de $20 \%$ en 1982 à $25,3 \%$ en 1999 . Ce taux a ensuite oscillé entre $20 \%$ et $24 \%$ dans les années 1990-2000, pour atteindre 23,4\% en 2004 (Alami, 2006). En Tunisie, ce taux est passé de 18,9\% en 1975 à 24,2 \% en 2005 (Gana, 2007). L’Algérie a continué, entre 
1960 et 1980, à avoir les taux les plus bas du monde, bien que l'éducation des filles fût en augmentation (Kateb, 2006). Selon Fatiha Talahite (2007), les élites socialistes algériennes ont accordé la priorité à l'emploi des hommes par une planification centralisée et un système de compétences qui ne valorise pas les savoirs traditionnels et les capacités des femmes. Soulignons cependant quelques actions volontaristes comme l'usine électronique de Sidi Bel Abbes, «fleuron de l'emploi féminin ouvrier ». Tout en repoussant l'entrée au travail des Algériennes, les élites ont fait de l'accès des femmes à l'éducation une priorité. La période de transition vers la libéralisation de l'économie s'est traduite par une hausse du taux d'activité chez les femmes, qui est passé de moins de 5,5\% en 1977 à près de $17 \%$ en 1998 (Talahite, 2007).

Si les taux d'activité ont évolué dans les pays du Maghreb, la structure de l'emploi féminin révèle, en revanche, une ségrégation horizontale traditionnelle concentrant les femmes dans des branches féminisées où la main-d'œuvre est jeune et peu qualifiée (textile, cuirs, agroalimentaire, habillement-confection, etc.), et une ségrégation verticale traduisant les difficultés d'accès aux emplois hautement qualifiés et aux postes de responsabilité (Alami, 2006). L'évolution des taux d'emploi chez les femmes est paradoxalement parallèle à l'augmentation du chômage parmi celles-ci, et particulièrement parmi les diplômées. Au Maroc, en milieu urbain, il est passé de $9 \%$ en 1980 à 21,5\% en 2000; il a oscillé depuis, atteignant 19,8\% en 2004 (Alami, 2006). En Algérie, le chômage touchait $31 \%$ de la population active en 2001 (Talahite, 2007). En Tunisie, il était de $16,9 \%$ en 2004. Force est de constater qu'il frappe des catégories jusque-là épargnées, comme les diplômées de l'enseignement supérieur. En 2002, au Maroc, le chômage touchait 34,9 \% des diplômées de l'enseignement supérieur en milieu urbain, $31,1 \%$ de celles du niveau moyen et 11,6 \% des femmes sans diplôme (Alami, 2006).

En Turquie, les femmes sont plus présentes qu'au Maghreb dans le monde du travail, avec un taux d'emploi de près de $27 \%$ en 2015 . Cependant, il n'y a pas de loi contre la discrimination de genre à l'embauche ni pour l'égalité salariale. Ainsi, le revenu annuel d'une femme diplômée du niveau supérieur est de 1,3\% moindre que celui d'un homme ayant les mêmes compétences; $45 \%$ des travailleuses ne sont pas déclarées et elles connaissent les affres du secteur informel.

En contexte de crise économique, le travail à domicile, une réalité ancienne dans les différents pays étudiés, reprend du poids dans l'activité des femmes et il se confond avec le secteur informel. Au Maroc, le travail à domicile (Centre d'études et de recherches démographiques [CERED], 1995) et les emplois d'aide familiale (Direction de la statistique, 2002) représentent $52,5 \%$ des femmes actives contre $22,2 \%$ pour les hommes. Ces taux sont encore plus significatifs en milieu rural puisque les femmes, présentes dans l'artisanat, les travaux de la laine, la poterie, etc., ne sont pas toujours rémunérées. Le secteur informel devient finalement le terme générique qui englobe à la fois le travail à domicile et le travail indépendant des femmes. La flexibilité de la main- 
d'œuvre et la compression des coûts du travail qui caractérisent ce secteur font des travailleuses une sorte de variable d'ajustement et d'armée de réserve. À ce propos, Bruno Lautier (1994) a mis en évidence l'articulation entre l'économie informelle et les besoins de flexibilité prônée par la pensée néolibérale, qui l'envisage comme un facteur d'autonomie des populations dans la création de leurs emplois, ce qui désengage l'État et solutionne les problèmes de développement. L'apologie de la flexibilité participe de la déréglementation en matière sociale et elle fait prendre pour de la modernité ce qui n'est que le retour à la violence primitive du marché (Lautier, 1994 : 104).

Envisager le travail des femmes comme un véritable processus d'autonomisation et d'émancipation suppose nécessairement de renverser la tendance à sa précarisation et à son «informalisation », pour valoriser le salariat et la formation. La mise en place de politiques d'encouragement, comme la multiplication de structures dédiées à la petite enfance (crèches, jardins d'enfants, classes de maternelle, etc.) se révèle être cruciale, à condition que ces efforts aillent dans le sens de la valorisation de la place des femmes dans la société, par la promotion de leurs droits dans la famille et dans l'espace public.

\section{Où en est-on aujourd'hui?}

Les conflits armés et la montée de l'extrémisme religieux : des facteurs de régression Dans les pays étudiés, deux facteurs contribuent fortement à la régression du mouvement féministe et des droits des femmes. Le premier a trait à la géopolitique et aux conflits armés, qui ont des incidences directes sur les femmes et les enfants, victimes les plus vulnérables des guerres, des conflits et des problèmes économiques, sociaux et culturels qui en résultent. C'est le cas des Palestiniennes, des Syriennes, des Irakiennes et des Yéménites, qui ont vu leurs droits reculer à cause des guerres. Les conditions de vie des Irakiennes se sont dégradées pendant la guerre irano-irakienne et celle du Golfe, mais aussi au cours de l'embargo (1991-2003). La situation économique, sécuritaire et sanitaire a conduit à l'augmentation du taux d'illettrisme, à la malnutrition, au non-accès aux soins, et à la multiplication des viols, des enlèvements, de la traite des femmes et des meurtres au nom de la morale religieuse. Les Palestiniennes, par exemple, sont aux prises à la fois avec l'occupation israélienne, qui constitue un frein pour le mouvement des femmes (restriction de leur liberté d'action et de leur mobilité), et avec l'hostilité des groupes islamistes.

Le second facteur de régression correspond à la montée de l'extrémisme religieux, pour lequel la question féminine est l'une des principales cibles. On assiste, en effet, depuis plusieurs années, à une dynamique de "réislamisation» des sociétés du Machrek, du Maghreb et de la Turquie, qui engendre un repli sur les valeurs conservatrices et qui restreint les libertés des femmes. L'islamisme instrumentalise la question féminine pour en faire la clé et l'emblème de la « réislamisation», il rejette les droits des femmes et il renvoie ces dernières à leur rôle traditionnel. Particulièrement dans le monde arabe, les pouvoirs en place, qui instrumentalisent tout autant cette question, ont oscillé entre l'encouragement de la protestation féminine, sur laquelle ils s'appuient pour contrer 
l'opposition islamiste, et les concessions fréquemment faites à cette dernière, au détriment des femmes. Malgré un contexte défavorable, de nombreuses femmes poursuivent leur combat pour dénoncer la régression de leurs droits et elles mettent en place des stratégies multiples d'inclusion et de conquête du champ politique. Certaines ont pu accéder à la sphère publique par leur participation à la vie associative ou partisane, où elles expriment leurs opinions, leurs besoins particuliers et leurs problèmes, tandis que la majorité d'entre elles agissent quotidiennement pour trouver ou affirmer leur place dans le monde du travail et dans l'espace public. Ces femmes ont pris part à la contestation politique, défiant de nombreux interdits et tabous, comme le montre leur mobilisation lors des soulèvements arabes de 2011.

L'émergence de l'islam politique et/ou radical dans les trois pays du Maghreb a eu une incidence considérable sur le mouvement des femmes et sur leurs revendications, avec certaines distinctions, toutefois, qui méritent notre attention. Dans les années 1990, l'Algérie a dû composer avec une guerre civile meurtrière qui a d'abord opposé les groupes islamistes armés au pouvoir, puis à l'ensemble de la population (Belalloufi, 2012 : 242). Les associations féministes, traitées par les islamistes radicaux « d'éperviers du colonialisme de l'Occident» (Madani, 1990), n'avaient pas cessé de dénoncer les actes de violence et le discours liberticide des islamistes radicaux visant les femmes. Pendant la guerre civile, elles n'ont pas échappé aux massacres collectifs, aux enlèvements, aux viols et à l'esclavage dans les maquis des groupes armés islamistes, ni aux assassinats ciblés (militantes, femmes de l'armée et de la gendarmerie, enseignantes, étudiantes, lycéennes, écolières, cadres, médecins, artistes, etc.) (Semmoud, 2016). L'ensemble de la population étant touché, les formes de résistance, les manifestations et les actions de dénonciation se sont multipliées, malgré la terreur. Cette tragédie a paradoxalement permis au mouvement féministe algérien d'élargir sa base à des femmes de catégories diverses qui s'opposaient à l'islamisme radical meurtrier et qui puisaient la notion d'égalité dans leur vécu dramatique pendant la guerre civile.

Si la tendance du mouvement féministe en Tunisie et en Algérie reposait sur une conception universaliste, la démarche au Maroc était « réformiste», suivant une relecture féministe de l'histoire de l'islam dont la figure de proue était Fatima Mernissi. Aujourd'hui, plusieurs conceptions cohabitent dans les trois pays, avec des convergences quant aux revendications essentielles. L'analyse de Houria Alami M'Chichi (2014) sur le Maroc oppose le mouvement féministe, porté par des intellectuelles de gauche, aux autres formes de mobilisation des femmes, notamment dans des organisations mixtes où les revendications générales sont censées défendre leurs droits. Des dynamiques d'émancipation aux temporalités différentes sont à l'œuvre dans les deux cas.

Les possibilités pour les femmes de faire valoir leurs droits au sein de structures mixtes de l'islam politique nous semblent vaines. En Algérie, une section féminine du Hamas (islamistes modérés) a été créée en 1989 en opposition au mouvement féministe. Le discours idéologique des dirigeantes s'appuyait sur le projet de société islamique, dans 
lequel le rôle des femmes était restreint à la famille. Outre la revendication de l'application de la charia et de la non-mixité dans les transports, à l'école et au travail, l'action de cette association reste caritative. En Tunisie, les associations islamistes rejettent le projet moderniste et le féminisme d'État, qu'elles considèrent comme étant peu conformes à l'identité arabo-musulmane, et elles font du prosélytisme auprès des femmes et des familles. Dans tous les cas, c'est une voie qui éloigne les femmes du pouvoir d'agir et de l'empowerment, seules perspectives permettant une réelle émancipation.

En Turquie, l'évolution du mouvement des femmes ne s'est pas non plus effectuée d'une manière univoque et la montée de l'islam politique, dans les années 1990, a également fait obstacle à cette évolution. Critiquant la subordination des femmes dans la hiérarchie des partis islamistes, un courant de musulmanes féministes s'est formé pour protester contre le fait que les militantes étaient exclues des responsabilités politiques et les femmes, mises à l'écart des processus socioéconomiques. Ce courant s'est mobilisé pour rehausser la place des femmes dans la société musulmane, mais aussi pour contrer l'interdiction du port du voile dans les universités turques. Cette dernière mobilisation a été fortement instrumentalisée par des partis comme le Parti de la prospérité, qui représentait l'islam politique dans les années 1990 et qui est devenu ensuite le Parti de la justice et du développement dirigé par Recep Tayyip Erdoğan. La lutte des femmes en Turquie s'est donc progressivement scindée en deux, entre celles qui revendiquaient leurs droits dans un système laïc et celles qui les réclamaient dans le cadre des valeurs islamiques et de l'équilibre entre les sexes.

Les chemins de l'émancipation apparaissent donc variés et ils empruntent des détours plus ou moins longs. En outre, bien qu'elles soient diabolisées par les forces conservatrices dans le monde arabe et en Turquie, les idées et les valeurs du mouvement féministe moderniste réussissent tant bien que mal à imprégner plus largement les revendications des femmes. Debbie Cameron et Joan Scanlon (2014) définissent le féminisme « [...] comme une reconnaissance du fait que les femmes sont opprimées et comme un engagement à changer cet état de fait. Par-delà cette définition, on peut avoir toutes sortes de différences d'opinions quant au pourquoi de l'oppression des femmes et toutes sortes de points de vue différents quant à des stratégies pour transformer cette situation » (Cameron et Scanlon, $2014: 86$ ).

Ainsi, la posture de ces auteures nous semble représenter la réalité mouvante et diverse du mouvement des femmes dans les pays musulmans.

\section{Focalisation naturelle des luttes contre la violence}

Outre la brutalité des lois discriminatoires, les femmes subissent des violences familiales comme les crimes d'honneur, le mariage précoce et forcé, et l'excision des filles. Malgré l'organisation de campagnes officielles de sensibilisation auprès de la population dans plusieurs pays (Syrie, Jordanie, Liban, etc.), des femmes sont encore régulièrement assassinées au nom de l'honneur et les meurtriers continuent à bénéficier d'allégements de peine. Elles subissent également d'autres formes de violence: l'une des plus intolérables socialement réside dans le climat d'insécurité et les situations de brutalité 
physique ou verbale auxquelles elles doivent faire face dans les lieux publics. La violence d'État s'est manifestée en Égypte par le recours, en 2011, à des tests de virginité sur de jeunes manifestantes détenues dans des prisons militaires afin de les réduire au silence. C'est pourquoi, dans ces différents pays, les luttes des féministes se sont focalisées sur le thème de la violence, en faisant pression sur les gouvernements afin de l'inscrire dans les préoccupations politiques. La Turquie constitue un exemple en la matière, car le mouvement féministe a réclamé la mise en place d'un arsenal juridique important qui criminalise diverses formes de violence, comme le harcèlement sexuel, la violence domestique ainsi que la violence physique et sexuelle.

Dans les différents pays étudiés, les femmes poursuivent également leurs mobilisations pour résister aux attaques des islamistes radicaux contre leurs droits et pour revendiquer auprès de leurs États l'adoption sans réserve de la Convention internationale sur l'élimination de toutes les formes de discrimination à l'égard des femmes (CEDEF). Elles restent présentes aussi dans les mouvements sociaux et les actions de résistance liés à leur quotidien, dans leurs milieux de travail et dans leurs quartiers. Elles participent aux luttes syndicales et sont parfois à l'origine de grèves, même si leur rôle dans le syndicat ne se traduit pas par des responsabilités au sein des instances. En Tunisie, elles représentent ainsi $48 \%$ de la base de l'Union générale des travailleurs tunisiens (UGTT), mais seulement $1 \%$ ont pu se hisser au sommet.

\section{Les réseaux nationaux et régionaux : une force}

La diversité du mouvement féminin n'a pas empêché la construction de synergies, de concertations, de solidarités et d'actions communes à différentes échelles, dans chaque pays, dans les régions, dans le monde arabe et sur la scène internationale. Le Maghreb illustre bien la constitution de réseaux qui fédèrent les efforts et brisent l'isolement, et le découragement des associations locales de les placer au cœur de dynamiques collectives fortes. Le collectif Hrayer Tounès (Femmes libres de Tunisie) rassemble des groupes d'horizons divers. On y trouve des associations et des structures «historiques » autonomes : l'Association tunisienne des femmes démocrates (ATFD), l'Association des femmes tunisiennes pour la recherche sur le développement (AFTURD), les commissions «femmes» du syndicat majoritaire, l'UGTT, et la Ligue tunisienne des droits de l'homme (LTDH). S'y joignent les groupes qui se sont démarqués du pouvoir comme l'Union nationale de la femme tunisienne (UNFT) ainsi que de nouveaux groupes comme le Collectif pour les femmes de Tunisie ou le réseau Doustourna. Le réseau Wassila, né en 2000, en Algérie, rassemble des associations féministes et des professionnelles de la santé, du travail social et du droit, qui conjuguent leurs efforts pour aider les victimes de violences. Au Maroc, le réseau Le printemps de la démocratie et de l'égalité, né en 2011 à l'occasion de la révision constitutionnelle, regroupe une vingtaine d'associations qui ont réussi à faire avancer le statut et les droits des femmes dans le projet de constitution. À l'échelle régionale, le Collectif Maghreb-Égalité 95 (CMÉ 95) est un réseau de militantes et d'intellectuelles féministes qui contribue à soutenir et à renforcer les revendications nationales en développant une solidarité régionale. La création de ces structures est parallèle à leur connexion avec des organisations non gouvernementales et 
des organismes internationaux qui soutiennent leurs activités, notamment par du financement.

Les nouveaux médias : espaces d'expression et de communication

Les femmes du Machrek, du Maghreb et de Turquie ont particulièrement investi Internet et les réseaux sociaux (Facebook, Twitter) pour communiquer et se mobiliser. Ces nouveaux médias sont ainsi devenus un outil important pour contourner les difficultés d'accès aux moyens d'expression traditionnels ou officiels et les obstacles à la parole dans l'espace public. La création de blogues répondant aux besoins d'expression variés des femmes et leur donnant la possibilité de l'anonymat, la diffusion de témoignages vidéo, le recours à Facebook et à toutes les autres formes de médias sociaux rendent les revendications et les luttes féminines visibles et suscitent le débat. Ces formes de communication et ces façons de se renseigner permettent non seulement de sensibiliser l'opinion publique aux problèmes des femmes, mais aussi de dénoncer ces problèmes pour changer le regard porté sur elles. Ces nouvelles technologies ont aussi mis en évidence la capacité des femmes à s'organiser en réseaux et à créer des actions militantes dans le but de s'approprier la rue, un lieu conçu comme un espace militant qui agit sur le changement social (Fortier et Monqid, 2017).

\section{Conclusion}

À l'issue de cette réflexion, nous proposons de conclure sur trois idées plus ou moins liées qui sont autant d'ouvertures au débat. En premier lieu, l'analyse des dynamiques d'émancipation des femmes au Machrek, au Maghreb et en Turquie ne peut faire l'impasse sur l'effet conjugué, d'une part, du retour en force d'une vision résolument conservatrice de l'islam et, d'autre part, d'un contexte fait généralement de tensions politiques et de guerres. Ces facteurs sont à l'origine de violences meurtrières, de dégradations brutales des conditions de vie et de régressions considérables sur le plan des droits de la personne, qui affectent plus fortement les femmes, dont beaucoup vivent des circonstances dramatiques (migration forcée, exil, situation de réfugiées). En même temps, ce contexte ne doit pas faire en sorte que les dynamiques d'émancipation des femmes dans ces régions deviennent un champ aveugle des réflexions parmi les organismes internationaux, les chercheurs et chercheuses, et surtout, le " "féminisme international" [...] appui majeur pour les mouvements de femmes locaux et nationaux qu'il contribue à orienter, en même temps qu'il s'en nourrit » (Bacqué et Biewener, $2013: 54)$.

En deuxième lieu, la logique néolibérale du développement des pays étudiés est aujourd'hui à l'origine de l'exacerbation des inégalités de sexe et d'un recul social et économique des femmes, qui ont été les premières victimes du chômage, du travail informel, des baisses arbitraires de salaire, etc. L'ouvrage de Marie-Hélène Bacqué et Carole Biewener (2013), qui articule le développement - notamment pour l'Asie du Sud - et l'empowerment des femmes, apporte un éclairage fécond. Les auteures passent en revue les diverses positions sur le rapport entre femmes et développement, et elles mettent en évidence les effets sur leur émancipation. En s'appuyant sur le texte d'Ester 
Boserup (1970), elles expliquent que les critiques formulées dans les années 1970 par rapport aux théories et aux politiques de développement en avaient souligné les effets négatifs, notamment la marginalisation des femmes, l'aggravation de leur situation d'infériorité et l'accroissement de leur pauvreté (Bacqué et Biewener, 2013 : 55).

Dans les années 1980, le renouvellement de cette analyse par le réseau Gender and Development va plus loin. Il introduit d'abord la distinction faite par les féministes entre le sexe comme catégorie biologique et le genre comme catégorie sociale. Il se démarque ensuite du « féminisme libéral », qui soutient une orientation individualiste sans remettre en cause les conditions structurelles de la domination en ce qui a trait au genre et à la classe, et qui conserve une vision eurocentriste du monde n'intégrant pas les perspectives non occidentales (Bacqué et Biewener, 2013: 56). Enfin, il met l'accent sur les structures et les dynamiques des relations de genre et, pour pouvoir les transformer, cherche à comprendre comment celles-ci se négocient. « La question de la redistribution du pouvoir est posée dans une approche qui appréhende la construction des inégalités à l'intersection des questions de genre, de classe, de race et des facteurs liés au colonialisme et au postcolonialisme. » (Bacqué et Biewener, 2013: 56) Ces points de vue résonnent comme un écho des perspectives et des démarches à privilégier pour analyser et faire évoluer la situation des femmes dans le monde arabe et musulman.

En troisième lieu, l'empowerment tel que l'analyse l'ouvrage de Bacqué et Biewener (2013) nous semble être un paradigme efficace à la fois pour comprendre les processus d'émancipation des femmes musulmanes, notamment les raisons des avancées, des reculs et des contradictions, et pour en préciser les conditions. Selon les auteures, « l'empowerment articule deux dimensions, celle du pouvoir (d'agir sur et avec) et celle du processus d'apprentissage pour y accéder» (Bacqué et Biewener, 2013: 6). Dans la lignée de Michel Foucault, la notion de pouvoir, celui exercé par ceux «d'en bas », est retravaillée et complexifiée, notamment par le mouvement féministe aux États-Unis. On distingue ainsi le «pouvoir intérieur» ou pouvoir de l'individu de s'affranchir de son aliénation; le "pouvoir de » ou pouvoir d'agir sur les transformations sociales; et le « pouvoir avec» ou pouvoir de l'individu à s'inscrire dans l'action collective. Parmi les conditions pour faire émerger l'empowerment, Bacqué et Biewener insistent d'abord sur la conscientisation, une approche mise au point notamment par Paulo Freire (1970), en Amérique latine, au sein du mouvement d'éducation populaire. L'objectif de Freire est de lutter contre l'aliénation des individus, ce qui passe d'abord par la transformation de l'image de soi pour créer une estime de soi. Ensuite, l'accent est mis sur le rôle des facilitators évoqués notamment dans le guide Violence Against Women: A Curriculum for Empowerment, Facilitator's Manual, paru en 1985. On peut considérer qu'au Machrek, au Maghreb et en Turquie, ces conditions sont plus ou moins réunies dans la vie associative, syndicale et partisane, et dans les réseaux nationaux et régionaux, mais aussi dans les nouveaux médias. Cependant, compte tenu du contexte évoqué précédemment, les processus d'apprentissage sont loin de toucher largement les femmes dans ces pays, et en particulier les plus marginalisées d'entre elles. 
Notes de bas de page

${ }^{1}$ Carte des pays étudiés.

${ }^{2}$ Mouvement de renaissance arabe.

3 À titre d'exemple, selon les données des Nations Unies (http://data.un.org), les taux nets de scolarisation au niveau secondaire, en 1984, étaient respectivement de $32 \%$ chez les filles et de 44,9\% chez les garçons. Entre 2009 et 2014, ils sont passés à $81 \%$ et $77 \%$. En Tunisie, en 1985, ils étaient de $26 \%$ chez les filles et de $38 \%$ chez les garçons. Entre 2009 et 2014, ils sont passés à $77 \%$ et $69 \%$.

${ }^{4}$ Selon les Nations Unies (http://data.un.org), les taux de prévalence des méthodes modernes de contraception, entre 2009 et 2014, étaient de $57 \%$ en Algérie, de $59 \%$ en Égypte, de $67 \%$ au Maroc, de $63 \%$ en Tunisie et de $73 \%$ en Turquie. À titre de comparaison, en France et au Canada, ils étaient respectivement de $76 \%$ et $74 \%$ pour la même période.

\section{Lexique des termes arabes}

Nahda: Terme qui signifie « renaissance » et qui est apparu dans la période de l'entredeux-guerres. L'écrivain Jurgi Zaydan (1861-1914) fut le premier à l'utiliser en référence à la Renaissance européenne (idée de renaissance et rupture avec l'obscurantisme du Moyen Âge).

An-nahda an-nisâ'iyya : Renaissance féminine.

Wafd : Terme qui signifie littéralement délégation. Il s'agit du plus ancien parti politique nationaliste égyptien.

Fatât ash-sharq: La fille de l'Orient.

Moudawana: Code de la famille marocain.

\section{Objectifs d'apprentissage}

- Avoir une meilleure connaissance de la condition des femmes et de leur mouvement au Machrek, au Maghreb et en Turquie.

- Permettre la compréhension de ces sociétés par le biais du genre.

- Connaître les voies précises de l'émancipation des femmes en contextes difficiles et les hybridations qu'elles opèrent en s'appropriant les modèles internationaux, en lien avec la réalité de leurs sociétés.

\section{Questions de réflexion}

- Quel est l'ancrage historique des dynamiques d'émancipation des femmes au Machrek, au Maghreb et en Turquie, et quels en sont les éléments déclencheurs?

- Quelles sont les conditions d'émergence des droits des femmes?

- Comment, face aux facteurs de régression (tensions politiques, guerres, montée du conservatisme islamiste), les femmes poursuivent-elles leur processus d'émancipation?

Suggestions de lecture 


\section{Turquie}

Başbuğu-Yaraman, Aysegül. 1996. «La femme turque dans son parcours émancipatoire (de l'Empire Ottoman à la République) ». Cahiers d'études sur la Méditerranée orientale et le monde turco-iranien $21: 15-32$.

Dedeoğlu, Saniye. 2010. «Visible Hands - Invisible Women: Garment Production in Turkey ». Feminist Economics 16(4) : 1-32.

Weibel, Nadine B. 1995. «Femmes de Turquie : entre sécularisation et religion ». Dans La place des femmes (coll.), 247-250. Paris : La Découverte.

\section{Maghreb}

Delphy, Christine et Amel Mahfoudh (coord. éditoriale). 2014. «Féminismes au Maghreb ». Nouvelles Questions Féministes 33(2). http://www.cairn.info/revuenouvelles-questions-feministes-2014-2.htm.

Thébaud, Françoise et Michelle Zancarini-Fournel. 2002. "CLIO, Histoire, Femmes et Sociétés : naissance et histoire d'une revue ». Clio, Femmes, Genre, Histoire 16 : 9-22. http://clio.revues.org/42

Machrek

Abu Lughod, Lila. 1998. Remaking Women: Feminism and Modernity in the Middle East. Princeton : Princeton University Press.

Al Ali, Nadje. 2000. Secularism, Gender and the State: the Egyptian Women's Movement. Cambridge : Cambridge University Press.

Arenfeldt, Pernille et Nawar Al-Hassan Golley. 2012. Mapping Arab Women's Movements: A Century of Transformations from Within. Caire : AUC Press.

Dupont, Anne-Laure et Catherine Mayeur-Jaouen (dir.). 2002. Débats intellectuels au Moyen-Orient dans l'entre-deux-guerres. Collection « Revue des mondes musulmans et de la Méditerranée », 95-98. Aix-en-Provence : Edisud.

\section{Ressources Internet}

Banque mondiale. Base de données Women, Business and the Law. http://wbl.worldbank.org/

Initiative pour le travail et l'embauche des femmes. http://www.keig.org/?lang=en.

\section{Bibliographie}


Alami M'Chichi, Houria. 2014. «Les féminismes marocains contemporains. Pluralité et nouveaux défis ». Nouvelles Questions Féministes 33(2) : 65-79.

Bacqué, Marie-Hélène et Carole Biewener. 2013. L'empowerment, une pratique émancipatrice? Collection « Politiques et sociétés ». Paris : La Découverte.

Belalloufi, Hocine. 2012. La démocratie en Algérie, réforme ou révolution? : Sur la crise algérienne et les moyens d'en sortir. Préface de Samir Amin et postface de Sadek Hadjeres. Alger : Lazhari Labter Éditions/APIC Éditions.

Benadada, Assia. 1999. "Les femmes dans le mouvement nationaliste marocain ». Clio, Femmes, Genre, Histoire 9.

Bernard-Maugiron, Nathalie. 2017. «Le statut juridique des femmes dans l'Égypte postrévolutionnaire ». Dans Corps des femmes et espaces genrés arabo-musulmans. Sous la direction de Corinne Fortier et Safaa Monqid, 93-103. Paris : Karthala.

Boserup, Ester. 1970. Woman's Role in Economic Development. Londres : Earthscan.

Cameron, Debbie et al. 2014. «Convergences et divergences entre le féminisme radical et la théorie queer ». Nouvelles Questions Féministes 33(2) : 80-94.

CERED. 1995. Enquête nationale sur la famille. Rabat : Centre d'études et de recherches démographiques (CERED).

Deniz, Kandiyoti. 1998. « Gender, Power and Contestation: Rethinking "Bargaining with Patriarchy" ». Dans Feminist Visions of Development: Gender Analysis and Policy. Sous la direction de Cecile Jackson et Ruth Pearson, 135-151. Londres : Routledge.

Direction de la statistique du Haut commissariat au Plan. 2017. http://www.hcp.ma/Direction-de-la-statistique a716.html

El Korso, Malika. 1998. «La mémoire des militantes de la Guerre de libération nationale ». Insaniyat $3: 25-51$.

Freire, Paulo. 1970. La pédagogie des opprimés. Paris : Maspero.

Gana, Alia. 2007. « Processus de libéralisation et dynamiques de l'emploi des femmes en Tunisie ». Autrepart 43(3) : 57-72.

Gerel, Sema et Kerem Karaosmanoğlu. 2006. «A new Islamic Individualism in Turkey: Headscarved Women in the City ». Turkish Studies 7(3) : 473-488. 
Jasser, Ghaïss et al. 2016. «Les luttes des femmes arabes contre le patriarcat, les pouvoirs tyranniques, l'islamisme, le colonialisme et le néocolonialisme ». Nouvelles Questions Féministes 35(2) : 6-16.

Kateb, Kamel. 2005. École, population et société en Algérie. Paris : L’Harmattan.

Lautier, Bruno. 2004. L'économie informelle dans le tiers monde. Collection « Repères ». Paris : La Découverte.

Lavoix, Valérie. 2010. «Femmes, pouvoir et voile en Syrie ». Hérodote 136(1) : 100120.

Lefebvre, Henri. 1970. La Révolution urbaine. Collection « Idées ». Paris : Gallimard.

Madani, Abassi (dirigeant de l'ex-Front islamique du salut). Entrevue avec l'AlgérieActualité le 4 janvier 1990.

Mahfoudh, Amel et Christine Delphy. 2014. « Entre dictatures, révolutions et traditions, la difficulté d'être féministe au Maghreb ». Nouvelles Questions Féministes 33(2) : 4-12.

Mahfoudh, Dorra et Amel Mahfoudh. 2014. « Mobilisations des femmes et mouvement féministe en Tunisie ». Nouvelles Questions Féministes 33(2) : 14-33.

Mejjati Alami, Rajaa. 2006. «Femmes et marché du travail au Maroc». L'Année du Maghreb I : 287-301.

Saadi, Nouredine. 1991. La femme et la loi en Algérie. Casablanca : Éditions Le Fennec.

Shaarawi, Huda. 1986. Harem Years: The Memoirs of an Egyptian Feminist, 1879-1924. Traduction et introduction par Margot Badran. Londres : Virago.

Semmoud, Nora. 2016. «Les femmes face à l'islamisation de la société. Chronique des années de braise: l'Algérie depuis 1988 ». Dans Femmes, printemps arabes et revendications citoyennes. Sous la direction de Gaëlle Gillot et Andrea Martinez, 113138. Marseille : IRD Éditions.

Talahite, Fatiha. 2007. "Algérie. L’emploi féminin en transition ». Communication au huitième Mediterranean Social and Political Research Meeting, Florence, Montecatini. 
